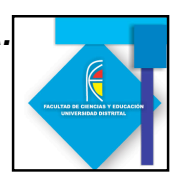

\title{
ANÁLISIS DEL SEGUNDO POSTULADO DE LA TEORÍA RELATIVISTA
}

\section{SECOND POSTULATE ANALYSIS OF RELATIVISTICTHEORY}

\author{
Andrés Yesid Sierra Pareja ${ }^{1}$
}

\section{Resumen}

En la enseñanza de la teoría de la relatividad se han evidenciado algunas dificultades en la comprensión del segundo postulado por parte de los estudiantes, lo que dificulta el apropiado desarrollo en la enseñanza de la teoría relativista en su totalidad; por consiguiente los estudiantes solo se han limitado a manejar la parte matemática, omitiendo la parte conceptual de la teoría.

Por tanto para el desarrollo de este trabajo se analizara el segundo postulado de la relatividad desde los diferentes puntos de vista descritos en la literatura y sus diferentes presentaciones, observando el papel que juega el postulado dentro de la teoría relativista.

Para cumplir con el objetivo del trabajo se seleccionarán y examinaran diferentes libros y artículos que divulguen la teoría relativista, desde los textos de la enseñanza de la física para los niveles de educación media y universitaria, libros histórico-filosóficos, textos de divulgación de la teoría e introductorios.

Palabras clave: Espacio, tiempo, espacio-tiempo, causalidad, simultaneidad, velocidad máxima, Velocidad de la luz, velocidad de información, marcos de referencia, ondas, medio de propagación, comprensión.

\section{Abstract}

In the teaching of the relativity theory have shown some difficulties in understanding the second postulate by students, which hampers the proper development in the teaching of the relativity theory its entirety, therefore students only have limited to handle the mathematics, the theory 's omitting the conceptual part.

Therefore, for the development of this work will analyze the second postulate of relativity from different points of view in the literature and its various presentations, looking at the role played by the postulate in relativity theory.

To meet the objective of the work will be selected and examined books and articles to disclose the relativistic theory from the texts of teaching physics to high school levels and university, historical and philosophical books, factual texts of theory and introductory.

\footnotetext{
${ }^{1}$ Universidad Pedagógica Nacional slrzog@hotmail.com
} 


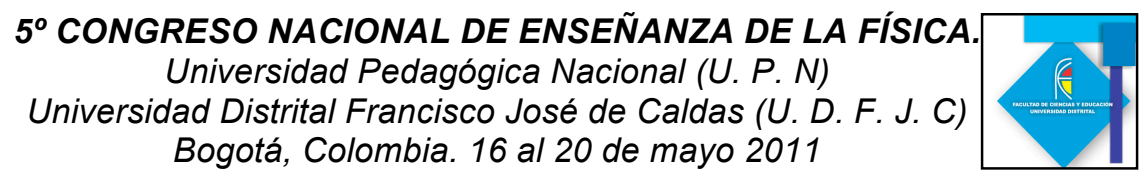

Keywords: Space, time, space-time, causality, simultaneity, Maximum speed, Speed of light, frameworks, waves, understanding, Keywords

\section{Introducción}

El análisis de textos permite evidenciar que tipo de información se está transfiriendo a determinada comunidad. Por esto, es importante analizar como los textos de física presentan y explican el segundo postulado de la relatividad especial, principalmente, aquellos textos que son la base para el desarrollo de conocimiento de universitarios con alguna relación a la física moderna.

Esta primera etapa del trabajo, estará dirigida hacia la indagación y análisis de textos, cuyos contenidos expresen el desarrollo de la TER, enfocándonos principalmente en la manera como dan a conocer, explican y ligan a la esta teoría.

Con base a lo anterior, se pretende desarrollar un primer lugar, un análisis que permita evidenciar dificultades y/o fortalezas en los textos, teniendo en cuanta las consideraciones anteriores, $y$ en segundo lugar, poder desarrollar un camino que me aproxime a desarrollo conceptual y a la comprensión de dicho postulado.

\section{Marco teórico}

Para el desarrollo del trabajo, se analizaran tres textos de enseñanza de la física de nivel universitario, dos textos de tipo histórico-filosóficos de la TER y dos textos de divulgación de la TER.

Enseñanza de la física de nivel universitario: Física para ciencias e ingeniería, Quinta Edición, Tomo II. Raymond A. Serway Robert J y Beichner, Física para la ciencia y la tecnología, Tercera Edición, Tomo II. Paul A. Tipler y Física M. Alonso, E.J Finn.

Histórico-filosófico: Introducción a la relatividad de Paul Langevin.

Divulgación: Que es la teoría de la relatividad de L. Landau, Y. Rumer y Cosmos de Carl Sagan.

La selección de los textos se debe a la gran demanda que presentan por los estudiantes y a recomendaciones por parte la profesora María Mercedes Ayala, quien actualmente en mi asesora de trabajo de grado. En estos textos, se revisara el capítulo de la teoría de la relatividad especial, enfocándonos en la presentación del enunciado del segundo postulado de dicha teoría.

\section{Textos de enseñanza de la física de nivel universitario}

\section{Enunciados del segundo postulado}

Texto Tipler: La velocidad de la luz es independiente de la velocidad de la fuente. (Alternativo) Todo observador mide el mismo valor c para la velocidad de la luz

Texto Serway: La constancia de la rapidez de la luz: la rapidez de la luz en el vacío tiene el mismo valor, $c=3.00 \times 10^{8} \mathrm{~m} / \mathrm{s}$, en todos los marcos inerciales, independientes de la velocidad del observador o de la velocidad de la fuente que emite la luz.

Texto Alonso: La velocidad de la luz es un invariante físico, y tiene el mismo valor para todos los observadores que estén en movimiento relativo uniforme. 
$5^{\circ}$ CONGRESO NACIONAL DE ENSEÑANZA DE LA FÍSICA.

Universidad Pedagógica Nacional (U. P. N)

Universidad Distrital Francisco José de Caldas (U. D. F. J. C)

Bogotá, Colombia. 16 al 20 de mayo 2011

\section{Explicación del texto}

Tipler:

El segundo postulado describe una propiedad común de a todas las ondas. La velocidad de una onda solo depende del medio de propagación. Se presenta de forma alternativa otro enunciado para la presentación de este: Todo observador mide el mismo valor c para la velocidad de la luz

Serway:

El segundo postulado es requerido por el primer postulado; si la velocidad de la luz no fuera la misma en todos los marcos inerciales, la medición de diferentes magnitudes de velocidad haría posible distinguir entre marcos inerciales, como resultado, se podría identificar un marco absoluto privilegiado, en contradicción con el primer postulado.

Alonso:

Los resultados negativos del experimento de Michelson y Morley llevaron a Einstein a descartar el concepto de la existencia de un éter. En su lugar propuso, como ley universal de la naturaleza que La velocidad de la luz es un invariante físico, y tiene el mismo valor para todos los observadores que estén en movimiento relativo uniforme.

\section{Análisis de la presentación del enunciado y explicación}

La idea principal es clara en los enunciados, la velocidad de la luz tiene el mismo valor para todos los observadores inerciales, independiente de la velocidad de la fuente o del observador. Sin embargo, el Serway y Alonso aportan dos ideas interesantes. Por ejemplo Serway indica la propagación de la luz en el vacío, y que la rapidez en este es, $c=3.00 \times 10^{8} \mathrm{~m} / \mathrm{s}$. Por otro lado, Alonso indica la velocidad de la luz como un invariante físico.

Dado que los textos de enseñanza se consideran una herramienta que facilita el proceso de enseñanza, se espera que estos aborden oportunamente una explicación lo suficientemente clara, para que el estudiante pueda comprender el postulado y entender cuál es la importancia de este dentro de la TER. Sin embargo dentro de los textos, solo se presenta el enunciado sin una justificación convincente. Por ejemplo, Tipler lo describe como el enunciado de una propiedad de todas las ondas, cosa que no permite entender la importancia de la luz frente a otro tipo de ondas y dentro de la teoría. Serway, por su parte, lo plantea como una exigencia del primer postulado. Al respecto surgen las siguientes preguntas ¿Acaso no son estos postulados independientes?, ¿Basta solo con el primero para fundamentar la TER?, ¿Por qué en el marco de la cosmovisión newtoniana es admisible el principio de relatividad galileano y el hecho de que la velocidad de una onda mecánica dependa del sistema de referencia desde la cual sea determinada? Así, las aclaraciones presentadas por estos autores no consiguen guiar al estudiante a comprender lo que este postulado implica, tan solo crea una imagen la cual debemos suponer que es correcta. Por ejemplo, indicar que la luz se comporta como una onda es correcto, pero ¿Qué tipo de onda es? ¿Qué tiene de especial ese tipo de ondas?, ¿Por qué este tipo de ondas se puede propagar en el vacio? y ¿Qué es el vacio? Sin embargo, restringir la velocidad de la onda tan solo al medio de propagación, puede ser incorrecto bajo ciertas consideraciones. Por ejemplo, si analizamos el caso de una onda sonora, la velocidad de dicha onda será la misma, si se analiza respecto al aire en reposo, ¿Qué ocurrirá si en el momento en que se produce la perturbación en el aire, hay un desplazamiento de esta, debido ráfaga de viento? esta cuestión, como muchas otras que se plantearan durante el desarrollo del trabajo, quedan en un primer momento abiertas en busca de respuestas.

Para finalizar este análisis, me basare en el carácter de invariante físico que le otorga el texto de Alonso a la velocidad de luz. Si bien, la velocidad de la luz es un invariante físico 


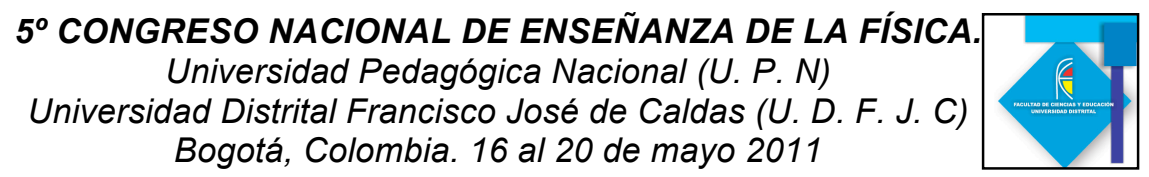

al cambiar de marco de referencia, no lo es al cambiar de medio de propagación, es decir, la sorprendente velocidad que desarrolla en el vacío, no será la misma el propagarse, por ejemplo, dentro de un fluido como el agua. Al igual que este, se podrían mencionar montones de medios de propagación, en los cuales se verá afectada la velocidad de la luz.

\section{Texto histórico-filosófico}

El texto de Langevin, Introducción a la Relatividad, no entra a especificar ni enunciar el segundo postulado, solo otorga indicios que permiten al estudiante enfocar sus estudios a determinado tema. Langevin analiza las ideas del espacio y el tiempo desde la mecánica racional y el electromagnetismo. Para desarrollar este análisis, trabaja la conexión entre eventos que ocurren en lugares diferentes desde las dos perspectivas -mecánica racional y la electromagnética-, desarrollando experimentos mentales con base a la velocidad de transferencia de información y la causalidad. En la mecánica racional, dos eventos son simultáneos independientes de la distancia en la que se encuentren, es decir, la velocidad de la señal que puede conectarlos, es infinita o instantánea, y con ello se deduce que su tiempo y espacio tienen un carácter absoluto. Para la concepción electromagnética, al identificarse una velocidad de información finita -que corresponde a la misma velocidad de la luz-, se pierde el sentido absoluto. En el marco de esta cosmovisión, que tiene como eje el concepto de campo se postula así que la información se trasmite de punto a punto de forma contigua, es decir que no lo hace de manera directa, y por lo tanto, su trasmisión o propagación gasta tiempo; concepción que se opone radicalmente a la newtoniana de acción directa y a distancia, que implica pensar en señales que conectan eventos en diferentes lugares que no gastan tiempo. Es decir, la perspectiva electromagnética del mundo exige pensar en una velocidad limite finita. Según el texto;

...Einstein fue el primero en demostrar cómo esta consecuencia de la teoría electromagnética basta para determinar los caracteres del espacio y del tiempo exigidos por una nueva concepción del universo. De acuerdo con lo que antecede, se concibe que la velocidad de la luz deba desempeñar un papel esencial en los enunciados nuevos: es la única velocidad que se conserva cuando se pasa de un sistema de referencia...

Con esta consideración se manifiesta un carácter no de espacio y tiempo, sino de un espacio-tiempo como un solo ente, además de indicar que es la única velocidad que se conserva al cambiar de marco de referencia, independiente de la velocidad y dirección de este.

\section{Textos de Divulgación}

En esta sección analizamos los textos, Que es la teoría de la relatividad de L. Landau, Y. Rumer, y Cosmos de Carl Sagan. En estos textos, se resalta la importancia de considerar la velocidad de la luz, como una ley de la naturaleza, debido a que es la velocidad máxima de envió de información, es decir, la velocidad de transmisión y recepción de señales, de dos marcos de referencia separados una distancia cualquiera, no puede ser infinita, esta no puede superar la velocidad máxima. Por ejemplo, en el texto de Landau se describe lo siguiente:

...las leyes de la naturaleza deben ser iguales en todos los laboratorios que se mueven unos respecto a los otros rectilínea y uniformemente. La afirmación de que ninguna velocidad puede superar el límite establecido es también una ley de la naturaleza y, por tanto, la magnitud de la velocidad máxima debe ser absolutamente igual en los diferentes laboratorios... 
$5^{\circ}$ CONGRESO NACIONAL DE ENSEÑANZA DE LA FÍSICA.

Universidad Pedagógica Nacional (U. P. N)

Universidad Distrital Francisco José de Caldas (U. D. F. J. C)

Bogotá, Colombia. 16 al 20 de mayo 2011

...la velocidad de la luz no es simplemente la velocidad de propagación de un fenómeno de la naturaleza. Esta velocidad, al mismo tiempo, juega el importantísimo papel de velocidad máxima...

Además el Cosmos añade:

...Para poder comprender el mundo, para evitar paradojas lógicas de este tipo al desplazamos a velocidades elevadas, hay que obedecer algunas reglas, algunos mandamientos de la naturaleza. Einstein codificó estas reglas en la teoría especial de la relatividad. La luz (reflejada o emitida) por un objeto se desplaza a idéntica velocidad tanto si el objeto se mueve como si está estacionario: No sumarás tu velocidad a la velocidad de la luz. Además, ningún objeto material puede desplazarse a velocidad superior a la de la luz: No te desplazarás a la velocidad de la luz ni a velocidad superior...

Por más que lo deseemos -actualmente- no nos es posible sobrepasar esta barrera cósmica. Nos acercaremos demasiado a tal barrera, pero nunca la igualaremos. La afirmación de no adicionar velocidades, en ambos textos, la justifican por medio de la observación y el comportamiento de experimentos-por ejemplo, de aceleración de partículas- suyos resultados tienden a la velocidad de la luz, mas nunca logran desarrollar dicha velocidad, sin importar que modificaciones se planteen en el experimento.

Para concluir esta sección, planteare la idea principal de los textos, e intentare desarrollar una pregunta, cuya respuesta espero poder dar con el tiempo, y el análisis. La velocidad de la onda luminosa, dependerá tan solo, del emisor de luz, no importa la dirección, ni su velocidad. Sin embargo, resulta interesante preguntarse, ¿Cuál será la velocidad de la luz, en un medio el cual se está desplazando?

\section{Conclusiones}

En este primer capítulo de exploración, tan solo podre describir conclusiones frente a las argumentaciones que presentan los libros, más no podre concluir la veracidad e importancia total, que posee ese segundo postulado.

Es un poco nostálgico, ver como los textos dedicados a la enseñanza de la física, quizás no consideran el problema de presentar una justificación adecuada al segundo postulado de la TER. Debido a esto, es vez de generar conocimiento puro, comprensible y admisible, crea soluciones facilistas, que no dejan ver el verdadero significado y sentido de las cosas. Con esto no quiero decir, que el libro deba desarrollar toda la teoría de la luz, pero sí, que mencione él ¿Por qué? Se hacen estas consideraciones, pero que estas no sean tan superficiales y además, que se le dé la importancia que tiene el postulado dentro de la teoría.

En cuanto al texto histórico-filosófico, alimenta la construcción de nuevas nociones, que son el desprendimiento de la mecánica racional a la nueva concepción electromagnética. Aunque no da razón precisa del postulado, se da cuenta que el desarrollo se da en el ámbito de las teorías electromagnéticas y de las teorías de la ondulación, por tanto podemos centrarnos en la indagación, comprensión y la relación que existe entre estas dos. Por otro lado, ubica el papel que desarrolla la velocidad de la luz dentro de la teoría, y como esta es la base para el desarrollo de nuevas nociones.

En los textos de divulgación, en primer lugar, es importante resaltar como intentan desvanecer las ideas del sentido común que rigen nuestro pensamiento por medio de los experimentos mentales, razonables y justificados si se considera la velocidad de la luz como velocidad limite; y en segundo lugar, el sentido e importancia que resaltan con gran determinación al indicar que esta es una ley de la naturaleza. 


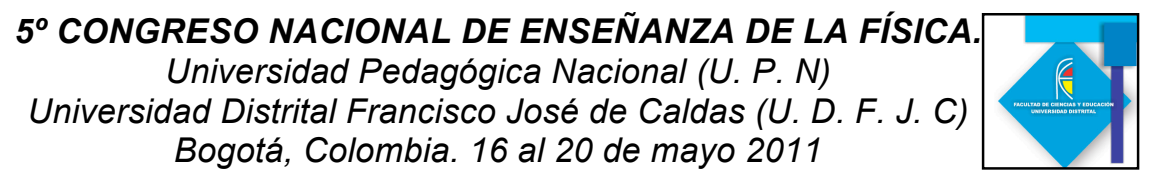

Por último, y a modo conclusión de este primer trabajo, se desean plantear las siguientes preguntas: ¿Qué tipo de onda es la luz?, ¿Qué característica contiene este tipo de ondas?, ¿Qué diferencia existe entre estas ondas y las ondas mecánicas?, ¿Por qué estas se pueden propagar en el vacio?, ¿qué es el vacio?, ¿Cuál es la influencia de la velocidad de la fuente respecto a las ondas?, cla medición de la velocidad de una onda mecánica se verá afectada, si por el medio en que se propaga tienen un desplazamiento debido a una corriente del mismo?, y ¿Cuál será el resultado si cambiamos la onda mecánica por una onda luminosa?, ¿Acaso no son los dos postulados independientes?, ¿Basta solo con el primero para fundamentar la TER?, ¿Por qué en el marco de la cosmovisión newtoniana es admisible el principio de relatividad galileano y el hecho de que la velocidad de una onda mecánica dependa del sistema de referencia desde la cual sea determinada?, ¿de qué depende y cuales son la características de la velocidad de la onda?, ¿Cómo sería la relatividad sin el segundo postulado?. Preguntas que espero guíen en la comprensión de el comportamiento de la luz.

\section{Referencias Bibliográficas}

Landau, L. y Rumer, R. (1985) Que es la teoría de la relatividad. Moscú: Mir. Sagan, C. (1980). Cosmos. España: Planeta.

Alonso, M y Finn, E.J. (1995). La teoría de la relatividad. Física (pp. 405-422). Estados unidos: Addison-Wesley Interamericana.

Langevin, P. (1956). La evolución del espacio y el tiempo. Introducción a la teoría de la relatividad (pp. 13-44). Buenos Aires: Ediciones Leviatán.

Tipler, P.A. (1993). Relatividad. Física para la ciencia y la tecnología, Tercera Edición, Tomo II (pp. 1099-1144). España: Reverte.

Serway, R.A. y Beichner, R.J. (2002). Relatividad. Física para ciencias e ingeniería, Quinta edición. Tomo II (pp. 1245-1288). México: Mcgraw-Hill Interamericana de México. 